\title{
Radiative electroweak penguins at LHCb
}

\author{
Albert Puig ${ }^{* \dagger}$ \\ École Polytechnique Fédérale de Lausanne \\ E-mail: albert.puig@epfl.ch
}

Radiative electroweak $B$ decays are sensitive probes of physics beyond the Standard Model. The latest results of the $B^{ \pm} \rightarrow K^{ \pm} \pi^{\mp} \pi^{ \pm} \gamma$ decays, performed using $3 \mathrm{fb}^{-1}$ of data collected in protonproton collisions with the LHCb detector, are presented. Approximately $14000 B^{ \pm} \rightarrow K^{ \pm} \pi^{\mp} \pi^{ \pm} \gamma$ signal events are reconstructed and selected, containing all possible intermediate resonances with a $K^{ \pm} \pi^{\mp} \pi^{ \pm}$final state in the $[1.1,1.9] \mathrm{GeV} / c^{2}$ mass range. The distribution of the angle of the photon direction with respect to the plane defined by the final-state hadrons is studied in intervals of $K^{ \pm} \pi^{\mp} \pi^{ \pm}$mass. The asymmetry between the number of signal events on each side of the plane is also studied, allowing to report the first direct observation of the photon polarisation in the $b \rightarrow s \gamma$ transition with a significance of $5.2 \sigma$.

The 15th International Conference on B-Physics at Frontier Machines at the University of Edinburgh, 14 -18 July, 2014

University of Edinburgh, $U K$

\footnotetext{
* Speaker.

${ }^{\dagger}$ on behalf of the LHCb collaboration.
} 


\section{Introduction}

Rare $b \rightarrow s \gamma$ flavour-changing neutral-current transitions are forbidden at tree level in the Standard Model (SM) and, as a consequence, are very sensitive to new physics (NP) effects arising from the exchange of new heavy particles in electroweak penguin diagrams. In such cases, the SM predicts that the emitted photon is predominantly left-handed since the recoil $s$ quark that couples to a $W$ boson is left-handed. However, in several NP models, such as the left-right symmetric model [1] or the minimal supersymmetric model (MSSM) [2], the photon can acquire a significant righthanded component. Although effects coming from NP are strongly constrained by measurements of inclusive radiative decays, there is still room for contributions that would enhance the right-handed photon polarisation component, as no experimental evidence for polarisation exists.

The radiative decay $B^{+} \rightarrow K^{+} \pi^{-} \pi^{+} \gamma$ was observed at the $B$ factories ${ }^{1}$ with branching fraction $\mathscr{B}=(27.6 \pm 1.8) \times 10^{-6}[3-5]$. Some exclusive decay modes with these final state particles have also been observed, such as $B^{+} \rightarrow K_{1}(1270)^{+} \gamma[6]$ or $B^{+} \rightarrow K_{2}^{*}(1430)^{+} \gamma$ [3,7], and upper limits have been set for decays through other resonances, such as $B^{+} \rightarrow K_{1}(1400)^{+} \gamma$ [6].

Here, the study of photon polarisation in the $B^{+} \rightarrow K^{+} \pi^{-} \pi^{+} \gamma$ decay is presented [8]. Information about the polarisation of the photon is obtained from its direction with respect to the normal to the plane defined by the momenta of the three final-state hadrons in their centre-of-mass frame. The up-down asymmetry between the number of events with the photon on either side of the plane is proportional to the photon polarisation $[9,10]$ and therefore measuring a nonzero asymmetry implies that the photon is polarised.

\section{Photon polarisation in $B^{+} \rightarrow K^{+} \pi^{-} \pi^{+} \gamma$ decays}

In this study, decays of the type $\bar{B} \rightarrow \bar{K}_{\text {res }} \gamma \rightarrow P_{1} P_{2} P_{3} \gamma$ are considered, where $\bar{K}_{\text {res }}$ is a kaon resonance and $P_{1}, P_{2}, P_{3}$ are three pseudoscalar mesons; for the $B^{+} \rightarrow K^{+} \pi^{-} \pi^{+} \gamma$ decay, $P_{1}=\pi^{+}$, $P_{2}=\pi^{-}$and $P_{3}=K^{+}$, while for the $B^{-} \rightarrow K^{-} \pi^{+} \pi^{-}$decay we have $P_{1}=\pi^{-}, P_{2}=\pi^{+}$and $P_{3}=K^{-}$. If we define the photon polarisation parameter $\lambda_{\gamma}$ as

$$
\lambda_{\gamma} \equiv \frac{\left|c_{\mathrm{R}}\right|^{2}-\left|c_{\mathrm{L}}\right|^{2}}{\left|c_{\mathrm{R}}\right|^{2}+\left|c_{\mathrm{L}}\right|^{2}}
$$

where $c_{\mathrm{L}}$ and $c_{\mathrm{R}}$ are the weak $\bar{B} \rightarrow \bar{K}_{\text {res }} \gamma$ amplitudes involving left- and right-handed photons, the fact that in the SM the photon from radiative $\bar{B}(B)$ decays is predominantly left- (right-) handed implies $\lambda_{\gamma} \simeq-1(+1)$, with corrections up to $10 \%[10,11]$.

Taking the final-state particle momenta $\left(\vec{p}_{i}\right.$ and $\left.\vec{p}_{\gamma}\right)$ in the $\bar{K}_{\text {res }}$ rest frame, and defining

$$
\cos \tilde{\theta} \equiv-\operatorname{sign}\left(s_{12}-s_{23}\right) \frac{\vec{p}_{\gamma}}{\left|\vec{p}_{\gamma}\right|} \cdot \hat{n}, \text { with } \hat{n} \equiv \frac{\vec{p}_{1} \times \vec{p}_{2}}{\left|\vec{p}_{1} \times \vec{p}_{2}\right|},
$$

where $s_{i j}$ is the invariant mass of the $P_{i} P_{j}$ system, the differential decay rate of $\bar{B} \rightarrow P_{1} P_{2} P_{3} \gamma$ going through a single resonance can be written using the helicity amplitude $\mathscr{J}_{\mu}$ as [10]

$$
\frac{\mathrm{d} \Gamma\left(\bar{B} \rightarrow \bar{K}_{\text {res }} \gamma \rightarrow P_{1} P_{2} P_{3} \gamma\right)}{\mathrm{d} s \mathrm{~d} s_{13} \mathrm{~d} s_{23} \mathrm{~d} \cos \tilde{\theta}} \propto|\overrightarrow{\mathscr{J}}|^{2}\left(1+\cos ^{2} \tilde{\theta}\right)+\lambda_{\gamma} 2 \operatorname{Im}\left[\vec{n} \cdot\left(\overrightarrow{\mathscr{J}} \times \vec{J}^{*}\right)\right] \cos \tilde{\theta},
$$

\footnotetext{
${ }^{1}$ The inclusion of charge-conjugate processes is implied throughout this document.
} 
where $s$ is the invariant mass of the $P_{1} P_{2} P_{3}$ system. In the case of overlapping intermediate resonances, one needs to consider their interference and Eq. 2.3 is not valid, leading to more complex dependencies on $\cos \theta$ [9]. However, even in the case of multiple resonances one can write the differential decay rate as a function of the even and odd powers of $\cos \tilde{\theta}$ and coefficients $a_{j}\left(s_{13}, s_{23}\right)$ :

$$
\frac{\mathrm{d} \Gamma\left(\bar{B} \rightarrow \bar{K}_{\text {res }} \gamma \rightarrow P_{1} P_{2} P_{3} \gamma\right)}{\mathrm{d} s \mathrm{~d} s_{13} \mathrm{~d} s_{23} \mathrm{~d} \cos \tilde{\theta}} \propto \sum_{j \text { even }} a_{j}\left(s_{13}, s_{23}\right) \cos ^{j} \tilde{\theta}+\lambda_{\gamma} \sum_{j \text { odd }} a_{j}\left(s_{13}, s_{23}\right) \cos ^{j} \tilde{\theta} .
$$

The structure of the decay rate can be exploited to study the photon polarisation. Then, the up-down asymmetry is defined as described in Ref. [9,12]:

$$
\mathscr{A}_{\mathrm{ud}} \equiv \frac{\int_{0}^{1} \mathrm{~d} \cos \tilde{\theta} \frac{\mathrm{d} \Gamma}{\mathrm{dcos} \tilde{\theta}}-\int_{-1}^{0} \mathrm{~d} \cos \tilde{\theta} \frac{\mathrm{d} \Gamma}{\mathrm{d} \cos \tilde{\theta}}}{\int_{-1}^{1} \mathrm{~d} \cos \tilde{\theta} \frac{\mathrm{d} \Gamma}{\mathrm{d} \cos \tilde{\theta}}}=C \lambda_{\gamma},
$$

where the constant $C$ takes into account the integral over the Dalitz plot, $a_{j}\left(s_{13}, s_{23}\right)$, and the angle $\cos \tilde{\theta}$. If $\mathscr{J}$ is known, $C$ can be calculated and this asymmetry allows the determination of the photon polarisation. However, in the case of $B^{+} \rightarrow K^{+} \pi^{-} \pi^{+} \gamma$, one has to take into account that different resonances in the $K^{+} \pi^{-} \pi^{+}$spectrum cannot be easily separated. Each of these resonances decays through multiple intermediate resonances contributing to the $K^{+} \pi^{-} \pi^{+}$final state, generating its own Dalitz plot structure in $s_{13}$ and $s_{23}$. Given the fact that the angular distribution of the photon depends on where you are in this Dalitz plot, with the additional complication of the presence of interference patters among resonances, any measurement needs to be performed inclusively.

Currently, it is not possible to determine the value of the photon polarisation from the inclusive up-down asymmetry due to the lack of precise theoretical calculations and of knowledge of the proportions between the different resonances in the studied region. However, a measurement of a non-zero up-down asymmetry would constitute a proof of photon polarisation.

\section{Event selection}

In this analysis, $p p$ collision data corresponding to an integrated luminosity of $3 \mathrm{fb}^{-1}$, recorded by the LHCb detector at $\sqrt{s}=7$ and $\sqrt{s}=8 \mathrm{TeV}$, have been used. First, three charged tracks with a minimum transverse momentum $\left(p_{\mathrm{T}}\right)$ of $0.5 \mathrm{GeV} / c$ and a total positive or negative unit charge are combined to form the $K$ resonance vertex. Track quality is ensured by requiring a good track $\chi^{2}$ fit and a low probability that the track is actually made of pseudorandom combinations of hits. The difference between the logarithms of the particle identification likelihoods for several hypotheses are used to identify the tracks as pions or kaons [13]. The vertex corresponding to the resonance is required to be isolated from other charged tracks in the event by comparing the $\chi^{2}$ of the three-track fit and the $\chi^{2}$ of all possible vertices that can be obtained by adding an extra track to the original vertex. The $K^{+} \pi^{-} \pi^{+}$mass is then required to be in the $[1.1,1.9] \mathrm{GeV} / c^{2}$ range. This three-hadron resonance is then combined with a photon with transverse energy larger than $3 \mathrm{GeV}$ to build the $B^{ \pm}$candidate. Anticoincidence with tracks pointing to the calorimeter is applied to distinguish neutral from charged electromagnetic particles and a multivariate tool based on cluster shape parameters is used to separate photons from $\pi^{0} \rightarrow \gamma \gamma$ in which the two photons are reconstructed as a single cluster in the calorimeter. Only candidates in the $4279-6829 \mathrm{MeV} / \mathrm{c}^{2}$ 

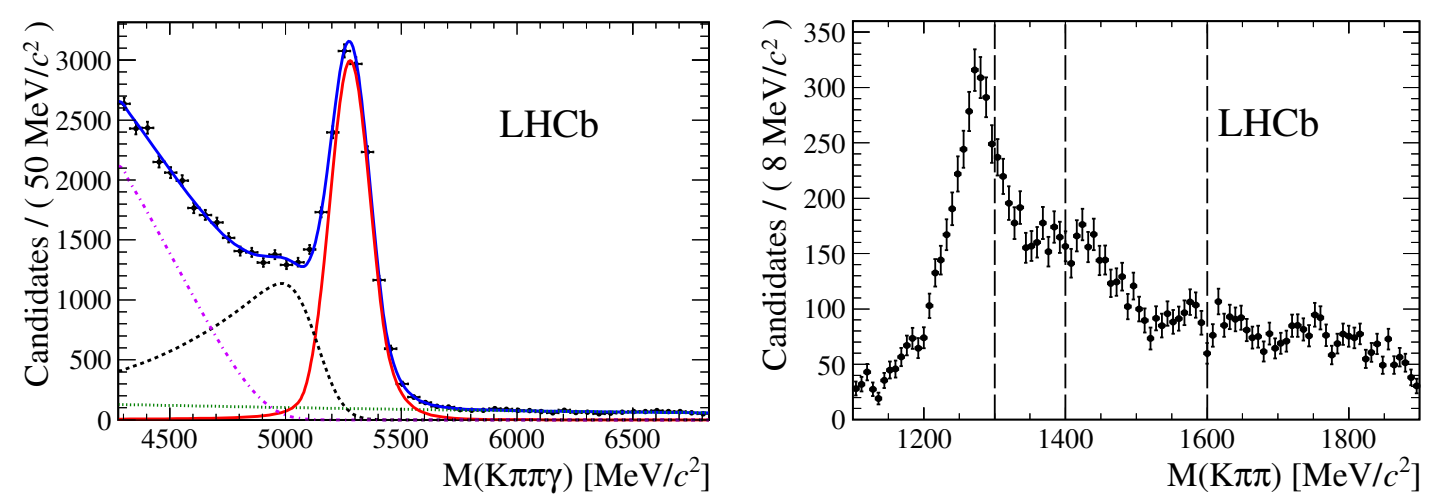

Figure 1: (left) Mass distribution of the selected $B^{+} \rightarrow K^{+} \pi^{-} \pi^{+} \gamma$ candidates, where the blue solid curve shows the fit results as the sum of signal (red solid), combinatorial background (green dotted), missing pion background (black dashed) and other partially reconstructed backgrounds (purple dash-dotted). (right) Background-subtracted $K^{+} \pi^{-} \pi^{+}$mass distribution of the $B^{+} \rightarrow K^{+} \pi^{-} \pi^{+} \gamma$ signal with the four intervals of interest separated by dashed lines.

invariant mass range are kept. Backgrounds that are expected to peak in this mass window, such as $B^{+} \rightarrow \bar{D}^{0} \rho^{+}$with $\bar{D}^{0} \rightarrow K^{+} \rho^{-}\left(\rightarrow \pi^{-} \pi^{0}\right)$ or $\rho^{+} \rightarrow \pi^{+} \pi^{0}$, are suppressed by removing all candidates consistent with having a $D^{0}$ or a $\rho^{ \pm}$meson in their decay chain. A boosted decision tree $[14,15]$ is used to further improve the separation between signal and background. Its training is based on the following variables: the impact parameter $\chi^{2}$ of both the $B^{ \pm}$and the final state hadrons, defined as the difference between the $\chi^{2}$ of a primary vertex (PV) reconstructed with and without the considered particle; the cosine of the angle between the reconstructed $B^{ \pm}$momentum and the vector pointing from the $\mathrm{PV}$ to the $B^{ \pm}$decay vertex; the flight distance of the $B^{ \pm}$meson; and the $K^{+} \pi^{-} \pi^{+}$vertex $\chi^{2}$.

\section{Fit description}

The mass distribution of the selected $B^{+} \rightarrow K^{+} \pi^{-} \pi^{+} \gamma$ signal is modeled with a double-tailed Crystal Ball [16] function, with a Gaussian core and power-law tails above and below the $B$ mass. Tail parameters are fixed from simulation and the width of the signal peak is fit separately for 2011 and 2012 data to account for differences in calorimeter calibration. Combinatorial and partially reconstructed backgrounds are considered in the fit, the former modeled with an exponential PDF, the latter described using an ARGUS function [17] convolved with a Gaussian function with the same width as the signal to account for the photon energy resolution. The contribution to the partially reconstructed background from events with only one missing pion is considered separately.

The fit of the mass distribution of the selected $B^{+} \rightarrow K^{+} \pi^{-} \pi^{+} \gamma$ candidates (Fig. 1) returns a total signal yield of $13876 \pm 153$ events, the largest sample recorded for this channel to date. Figure 1 also shows the background-subtracted $K^{+} \pi^{-} \pi^{+}$mass spectrum.

No peak besides that of the $K_{1}(1270)^{+}$resonance is clearly visible, but, as previously discussed, many kaon resonances are expected to contribute and interfere in the considered mass range [18]. The up-down asymmetry is thus studied inclusively in four theoretically-motivated intervals of $K^{+} \pi^{-} \pi^{+}$ mass [9]. The prominent $K_{1}(1270)^{+}$is isolated by choosing the $[1.1,1.3] \mathrm{GeV} / c^{2}$ interval. The 
$[1.4,1.6] \mathrm{GeV} / c^{2}$ interval, studied in Ref. [9], includes the $K_{1}(1400)^{+}, K_{2}^{*}(1430)^{+}$and $K^{*}(1410)^{+}$ resonances with small contributions from the upper tail of the $K_{1}(1270)^{+}$. The $[1.3,1.4] \mathrm{GeV} / c^{2}$ mass interval contains the overlap region between the two $K_{1}$ resonances, while the $[1.6,1.9] \mathrm{GeV} / c^{2}$ mass interval includes less known spin- 2 and spin-3 resonances.

In each of the four $K^{+} \pi^{-} \pi^{+}$mass intervals, a simultaneous fit to the $B$-candidate mass distribution in bins of $\cos \hat{\theta}(\cos \tilde{\theta}$ weighted by the $B$ candidate charge) is performed in order to determine the background-subtracted angular distribution. Each bin is fit with the previously described PDF, sharing all parameters apart from the signal and background yields. The resulting background-subtracted $\cos \hat{\theta}$ distribution, corrected for the selection acceptance, is fit with a fourth-order polynomial function normalized to unit area,

$$
f\left(\cos \hat{\theta} ; c_{0}=0.5, c_{1}, c_{2}, c_{3}, c_{4}\right)=\sum_{i=0}^{4} c_{i} L_{i}(\cos \hat{\theta}),
$$

where $L_{i}(x)$ is the Legendre polynomial of order $i$ and $c_{i}$ is the corresponding coefficient. With this model, the up-down asymmetry defined can be expressed as $\mathscr{A}_{\mathrm{ud}}=c_{1}-c_{3} / 4$.

\section{Results}

The fitted distributions in the four $K^{+} \pi^{-} \pi^{+}$mass intervals of interest are shown in Fig. 2 and summarized in Table 1. In order to illustrate the effect of the up-down asymmetry, the results of another fit imposing $c_{1}=c_{3}=0$, and thus removing the terms that carry the $\lambda_{\gamma}$ dependence, are overlaid for comparison.

The result is obtained from a $\chi^{2}$ fit of the normalized binned angular distribution, performed taking into account the full covariance matrix of the bin contents and all of the systematic uncertainties. These systematic uncertainties account for the effect of choosing a different fit model, the impact of the limited size of the simulated samples on the fixed parameters, and the possibility of some events migrating from a bin to its neighbor because of the detector resolution, the latter being the dominant contribution. The systematic uncertainty associated with the fit model is determined by performing the mass fit using several alternative PDFs, while the other two are estimated with simulated pseudoexperiments. Such uncertainties, despite being of the same size as the statistical uncertainty, do not substantially affect the fit results since they are strongly correlated across all angular bins.

The combined significance of the observed up-down asymmetries is determined from a $\chi^{2}$ test where the null hypothesis is defined as $\lambda_{\gamma}=0$, implying that the up-down asymmetry is expected to be zero in each mass interval. The corresponding $\chi^{2}$ distribution has four degrees of freedom, and the observed value corresponds to a $5.2 \sigma$ significance for nonzero up-down asymmetry.

\section{Conclusions}

The radiative $B^{+} \rightarrow K^{+} \pi^{-} \pi^{+} \gamma$ decay, with a $K^{+} \pi^{-} \pi^{+}$mass in the $[1.1,1.9] \mathrm{GeV} / c^{2}$ range, has been studied with a data sample corresponding to an integrated luminosity of $3 \mathrm{fb}^{-1}$ collected in $p p$ collisions at 7 and $8 \mathrm{TeV}$ centre-of-mass energies by the LHCb detector. A total of $13876 \pm 153$ signal events have been observed. 

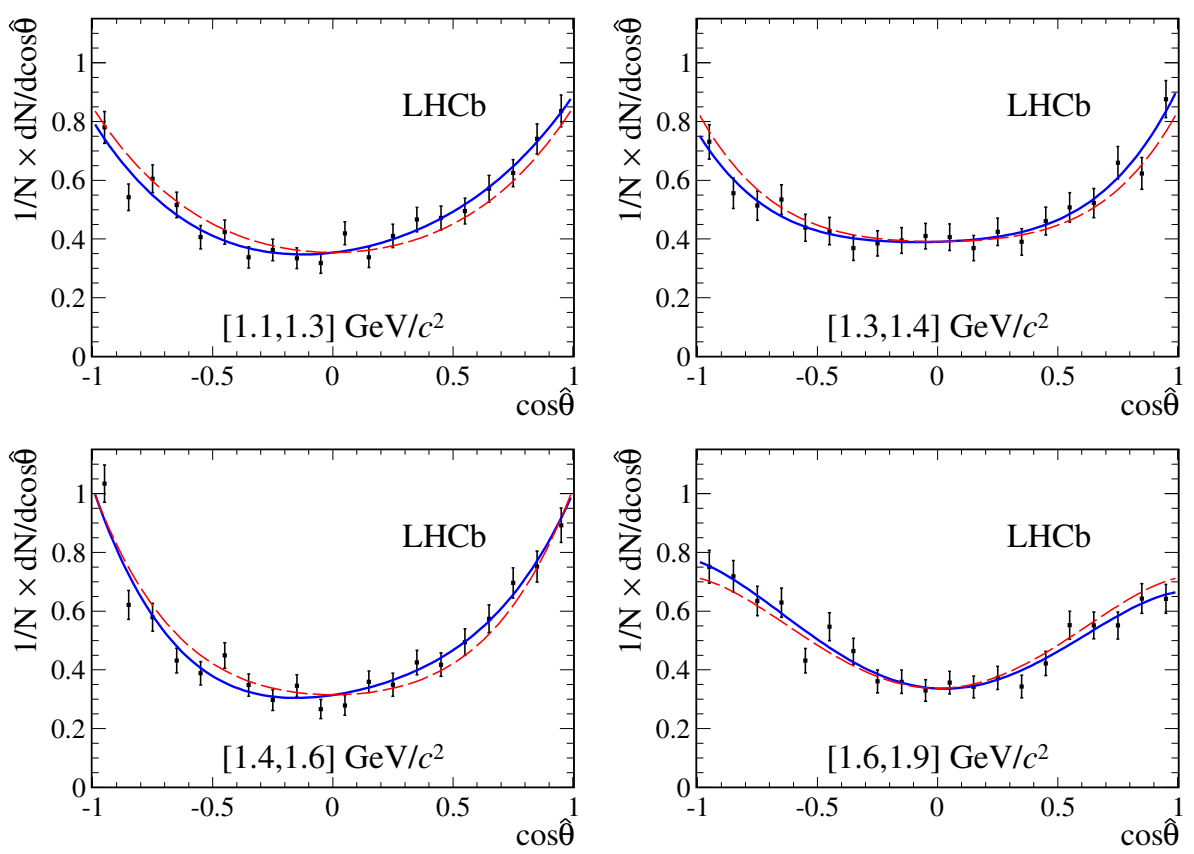

Figure 2: Distributions of $\cos \hat{\theta}$ for $B^{+} \rightarrow K^{+} \pi^{-} \pi^{+} \gamma$ signal in the four intervals of $K^{+} \pi^{-} \pi^{+}$mass. The solid blue (dashed red) curves are the result of fits allowing all (only even) Legendre components up to the fourth power.

Table 1: Legendre coefficients obtained from fits to the normalized background-subtracted $\cos \hat{\theta}$ distribution in the four $K^{+} \pi^{-} \pi^{+}$mass intervals, along with the corresponding up-down asymmetries. All the parameters are expressed in units of $10^{-2}$.

\begin{tabular}{lcccc}
\hline & {$[1.1,1.3] \mathrm{GeV} / c^{2}$} & {$[1.3,1.4] \mathrm{GeV} / c^{2}$} & {$[1.4,1.6] \mathrm{GeV} / c^{2}$} & {$[1.6,1.9] \mathrm{GeV} / c^{2}$} \\
\hline$c_{1}$ & $6.3 \pm 1.7$ & $5.4 \pm 2.0$ & $4.3 \pm 1.9$ & $-4.6 \pm 1.8$ \\
$c_{2}$ & $31.6 \pm 2.2$ & $27.0 \pm 2.6$ & $43.1 \pm 2.3$ & $28.0 \pm 2.3$ \\
$c_{3}$ & $-2.1 \pm 2.6$ & $2.0 \pm 3.1$ & $-5.2 \pm 2.8$ & $-0.6 \pm 2.7$ \\
$c_{4}$ & $3.0 \pm 3.0$ & $6.8 \pm 3.6$ & $8.1 \pm 3.1$ & $-6.2 \pm 3.2$ \\
\hline $\mathscr{A}_{\mathrm{ud}}$ & $6.9 \pm 1.7$ & $4.9 \pm 2.0$ & $5.6 \pm 1.8$ & $-4.5 \pm 1.9$ \\
\hline
\end{tabular}

The shape of the angular distribution of the photon with respect to the plane defined by the three final-state hadrons in their rest frame has been determined in four intervals of the $K^{+} \pi^{-} \pi^{+}$ mass spectrum. The up-down asymmetry, which is proportional to the photon polarisation, has been measured for the first time in each of these $K^{+} \pi^{-} \pi^{+}$mass intervals. From these four independent measurements, the first observation of nonzero photon polarisation in $b \rightarrow s \gamma$ transitions has been reported with a $5.2 \sigma$ significance level.

The reported photon angular distributions, including the values for the up-down asymmetry, may be used, if theoretical predictions become available, to determine for the first time a value for the photon polarisation, helping constrain the contribution of physics beyond the SM in the $b \rightarrow s \gamma$ sector. 


\section{References}

[1] J. C. Pati and A. Salam, Lepton number as the fourth color, Phys. Rev. D10 (1974) 275, Erratum-ibid. D11 (1975) 703; R. Mohapatra and J. C. Pati, A natural left-right symmetry, Phys. Rev. D11 (1975) 2558; R. N. Mohapatra and J. C. Pati, Left-right gauge symmetry and an isoconjugate model of CP violation, Phys. Rev. D11 (1975) 566; G. Senjanovic and R. N. Mohapatra, Exact left-right symmetry and spontaneous violation of parity, Phys. Rev. D12 (1975) 1502.

[2] L. L. Everett et al., Alternative approach to $b \rightarrow s \gamma$ in the uMSSM, JHEP 0201 (2002) 022, arXiv:hep-ph/0112126.

[3] Belle collaboration, S. Nishida et al., Radiative B meson decays into K $\pi \gamma$ and $K \pi \pi \gamma$ final states, Phys. Rev. Lett. 89 (2002) 231801, arXiv: hep-ex/0205025.

[4] BaBar collaboration, B. Aubert et al., Measurement of branching fractions and mass spectra of $B \rightarrow K \pi \pi \gamma$, Phys. Rev. Lett. 98 (2007) 211804, arXiv : hep-ex/ 0507031.

[5] Heavy Flavor Averaging Group, Y. Amhis et al., Averages of b-hadron, c-hadron, and $\tau$-lepton properties as of early 2012, arXiv:1207.1158, updated results and plots available at: http: //www.slac.stanford.edu/xorg/hfag/.

[6] Belle collaboration, H. Yang et al., Observation of $B^{+} \rightarrow K_{1}(1270)^{+} \gamma$, Phys. Rev. Lett. 94 (2005) 111802, arXiv:hep-ex/0412039.

[7] BaBar collaboration, B. Aubert et al., Measurement of the $B^{0} \rightarrow K_{2}^{*}(1430)^{0} \gamma$ and $B^{+} \rightarrow K_{2}^{*}(1430)^{+} \gamma$ branching fractions, Phys. Rev. D70 (2004) 091105, arXiv: hep-ex/ 0409035.

[8] LHCb collaboration, R. Aaij et al., Observation of photon polarization in the $b \rightarrow s \gamma$ transition, Phys. Rev. Lett. 112 (2014) 161801, arXiv:1402.6852.

[9] M. Gronau and D. Pirjol, Photon polarization in radiative B decays, Phys. Rev. D66 (2002) 054008 , arXiv:hep-ph/0205065.

[10] E. Kou, A. Le Yaouanc, and A. Tayduganov, Determining the photon polarization of the $b \rightarrow s \gamma$ using the $B \rightarrow K_{1}(1270) \gamma \rightarrow(K \pi \pi) \gamma$ decay, Phys. Rev. D83 (2011) 094007, arXiv: 1011.6593.

[11] B. Grinstein, Y. Grossman, Z. Ligeti, and D. Pirjol, The photon polarization in $B \rightarrow X \gamma$ in the standard model, Phys. Rev. D71 (2005) 011504, arXiv: hep-ph/ 0412019.

[12] M. Gronau, Y. Grossman, D. Pirjol, and A. Ryd, Measuring the photon helicity in radiative B decays, Phys. Rev. Lett. 88 (2002) 051802, arXiv: hep-ph/ 0107254.

[13] A. Powell et al., Particle identification at LHCb, PoS ICHEP2010 (2010) 020, LHCb-PROC-2011-008.

[14] L. Breiman, J. H. Friedman, R. A. Olshen, and C. J. Stone, Classification and regression trees, Wadsworth international group, Belmont, California, USA, 1984.

[15] R. E. Schapire and Y. Freund, A decision-theoretic generalization of on-line learning and an application to boosting, Jour. Comp. and Syst. Sc. 55 (1997) 119.

[16] T. Skwarnicki, A study of the radiative cascade transitions between the Upsilon-prime and Upsilon resonances, PhD thesis, Krakov, PL, Institute of Nuclear Physics, 1986, DESY F31-86-02, Appendix E.

[17] ARGUS collaboration, H. Albrecht et al., Search for hadronic $b \rightarrow$ u decays, Phys. Lett. B241 (1990) 278 .

[18] Particle Data Group, J. Beringer et al., Review of particle physics, Phys. Rev. D86 (2012) 010001. 